\title{
SIMPLE TWO-STEP COVALENT PROTEIN CONJUGATION TO PEG-COATED NANOCRYSTALS
}

\author{
S. Ya. PARYZHAK ${ }^{1}$, T. I. DUMYCH ${ }^{1}$, O. I. KARMASH ${ }^{1}$, E. E. BILA ${ }^{3}$, \\ D. STACHOWIAK ${ }^{2}$, M. BANSKI $I^{2}$, A. PODHORODECKI $I^{2}$, R. O. BILYY'四 \\ ${ }^{1}$ Danylo Halytsky Lviv National Medical University, Ukraine; \\ 凶e-mail: r.bilyy@gmail.com; \\ ${ }^{2}$ Wroclaw University of Science and Technology, Poland; \\ ${ }^{3}$ Ivan Franko Lviv National University, Ukraine
}

Covering of nanocrystals (NC) with a polyethylene glycol (PEG) envelop is a common way to increase their hydrophilicity, and compatibility with bio-systems, including increased retention time in the body. Colloidal semiconductor NC, also known as quantum dots (QD), particularly benefit from covering with PEG due to passivation of the inorganic core, while maintaining physical properties of the core. Despite many advantages of covering the surface with PEG, the covalent attachment of protein to hydroxyls of PEG is complicated. Here we propose a simple two-step approach for modification of PEG residues with subsequent covalent attachment of proteins. We were able to achieve specific NC targeting by means of attached protein as well as preserve their optical parameters (fluorescence intensity) in chemical reaction conditions. In the optimized protocol, ensuring removal of chemical byproducts by dialysis, we were able to omit the need for centrifugation (usually a limiting step due to particle size). The obtained NC-protein conjugate solutions contained $0.25 x$ of initial unmodified NC amount, ensuring a low dilution of the sample. During all reactions the $\mathrm{pH}$ range was optimized to be between 6 to 8 . The proposed approach can be easily modified for covalent targeting of different PEG-covered nanocomposites with proteins.

Ke y w o rd s: PEGylated nanocomposites, quantum dots, covalent protein conjugation.

$\mathrm{N}$ anocrystals (NC), particularly quantum dots (QD), are usually poorly compatible with bio-systems, being either toxic, hydrophobic, or unstable upon contact with components of living systems. Covering the NC with polyethylene glycol (PEG) usually overcomes these limitations, making PEGylation a popular modification approach. QD are a type of colloidal semiconductor NC that comprise a promising class of materials for labeling biological systems. Their nanoscale size is compatible with the majority of metabolic and internalization processes observed in cells $[1,2]$. Compared to organic fluorophores, QD show exceptional resistance to photodegradation and photobleaching, narrower photoluminescence with high quantum yield, broader absorption bands, larger effective Stokes shifts and higher absorption coefficients. In order to target QD to a specific mole- cule (e.g. proteins, peptides, organic and inorganic polymers, DNA, carbohydrates) that is present in the biological system under study, their surface must be chemically modified with organic substances. This procedure is called functionalization of nanoparticles and the resulting system may be considered a hybrid organic-inorganic nanocomposite [3], which possesses combined properties of both basic elements: the optical properties of the QD and the biological functionalities of organic molecules. These nanocomposites may be used as biolabels in monitoring biological processes at the molecular level.

Encapsulation of NC by a polymer, for example PEG, increases their water solubility and colloidal stability and reduces the cytotoxic effects. PEG is a polyether compound which is biologically inert, consisting of repeating ethylene ether units with linear or branched polyether $\left(\mathrm{HO}-\left(\mathrm{CH}_{2} \mathrm{CH}_{2} \mathrm{O}\right)-\mathrm{OH}\right)$ and is

(C) 2018 Paryzhak S. Ya. et al. This is an open-access article distributed under the terms of the Creative Commons Attribution License, which permits unrestricted use, distribution, and reproduction in any medium, provided the original author and source are credited. 
highly water soluble [4]. Despite many advantages of PEGylation, covalent attachment of protein to the hydroxyls of PEG is complicated.

There are many reports about covalent modification of PEG (for example by using carboxyl-PEGcarboxyl) [5] or using approaches to develop rapid PEG-based kits for covalent protein modification (for example, using bis-2-hydroxyethylglycinamide) [6].

The aim of the current work was to develop an effective and simple protocol for covalent and stable conjugation of protein(s) to PEG-coated NC.

\section{Materials and Methods}

Fluorescent microscopy was performed with a Carl Zeiss AxioImager A1 DIC/fluorescent microscope (Oberkochen, Germany) using a 1.3 NA 100× oil immersion objective. Fluorescent images were taken by a Zeiss AxioCam MRm III cooled digital CCD camera under constant exposure. Fluorescence of NC was evaluated at red channel $(610 \mathrm{~nm}$ emission was obtained at $532 \mathrm{~nm}$ excitation). Image analysis was performed using Fiji software (National Institutes of Health [NIH], Bethesda, MD, USA).

Human cervical adenocarcinoma HeLa cells were obtained from the American Type Culture Collection and cultured in RPMI-1640 and Eagle's minimum essential medium (EMEM) (SigmaAldrich Co., USA). The media were supplemented with $4 \mathrm{mM}$ L-glutamine, $10 \mathrm{mM}$ HEPES buffer, $100 \mathrm{U} / \mathrm{ml}$ penicillin, $100 \mu \mathrm{g} / \mathrm{ml}$ streptomycin (PAA, Pasching, Germany), and fetal calf serum (GibcoBRL, Eggenstein, Germany) at $10 \%$. Cultures were maintained under $5 \% \mathrm{CO}_{2}$ at $37{ }^{\circ} \mathrm{C}$. Cell necrosis was induced by incubation of cells, grown on slides, at $56{ }^{\circ} \mathrm{C}$ for $30 \mathrm{~min}$. ConA lectin was a kind gift of Prof. V. Antonyuk, Lectinotest laboratory, Ukraine. Incubation of cells with $\mathrm{NC}$ was done by addition of NC-PEG-CO-NH-ConA suspension to cells (final concentration $1 \% \mathrm{v} / \mathrm{v}$ ), incubating for $10 \mathrm{~min}$ at $37^{\circ} \mathrm{C}$ and imaging.

\section{Results and Discussion}

Reaction of a covalent protein attachment using zero-length cross-linker 1-ethyl-3-(3-dimethylaminopropyl)carbodiimide hydrochloride (EDC) is widely used in conjugation chemistry [7]. We also effectively used this approach to covalently attach proteins, particularly lectins, to inorganic rare-earth $\mathrm{NC}$ with subsequent tissue targeting via intravenous administration in animals [8-10]. Besides, the reaction produced no toxic byproducts, since EDC was transformed into a non-toxic urea derivative. Thus, we choose this approach for PEG modification. Both amino and carboxyl groups are available in protein molecules, allowing flexible options for conjugation. To convert the hydroxyl of the PEG residue into a carboxyl group to be further functionalized we used the reaction with monochloroacetic acid (MCAA), as previously described $[7,11]$. MCAA is a small molecule (MW 94,5), and the unreacted compound is effectively removed from reaction media by dialysis. The obtained carboxyl residue on PEG was conjugated to amine groups of proteins using EDC as a zero-length cross-linker, as described below.

As model NC we have selected core/shell CdSe/ CdS QD with a $5.2 \mathrm{~nm}$ diameter and a maximum of the first absorption band at $\lambda=570 \mathrm{~nm}$. These NC showed a maximum in fluorescence at $581 \mathrm{~nm}$ with $33 \mathrm{~nm}$ a full width at half maximum (FWHM), and were easily traceable under TexasRed filter using microscopy and 254/360 nm transilluminator during chemical modifications. As a model protein for conjugation we selected lectin ConA from Canavalia ensiformis, possessing specificity to glucosylated oligomannose residues found inside the cell [12], and also able to bind some surface glycans on specific immune cell subpopulations. HeLa cells used for testing do not express surface targets of ConA, thereby allowing a convenient testing of lectin-nanocomposite specificity.

Modification of NC-PEG with monochloroacetic acid (MCAA). The following protocol has been chosen as optimal for the modification of $\mathrm{NC}$ PEG with MCAA (schematically shown on Fig. 1, A):

1. We prepared reaction buffer $(\mathrm{RB})$, containing $1 \mathrm{M}$ MCAA in $3 \mathrm{M} \mathrm{NaOH}$ (important: use glass vials).

2. The conjugation buffer is quite reactive, thus we tested a few variations of conjugation with NCPEG, by mixing 1 volume of NC-PEG with:

- $1 / 4$ volume of $\mathrm{RB}$, providing $0.25 \mathrm{M}$ MCAA and $\mathrm{pH} \sim 12$.

- $1 / 10$ volume of $\mathrm{RB}$, providing $0.1 \mathrm{M}$ MCAA and $\mathrm{pH} \sim 10$.

- $1 / 100$ volume of $R B$, providing $0.01 \mathrm{M}$ MCAA and $\mathrm{pH} \sim 8$.

3. The obtained mixtures were incubated for $70 \mathrm{~min}$ at room temperature with intensive stirring.

4. The reaction was stopped by adding solid $\mathrm{NaH}_{2} \mathrm{PO}_{4}(4 \mathrm{mg} / \mathrm{ml})$ to neutralize the $\mathrm{pH}$ to $<6$, controlled by measuring $\mathrm{pH}$.

5. Excess reactants were removed by dialysis against $\mathrm{dH}_{2} \mathrm{O}$ overnight. Then the resulting volume 
was measured. The obtained product was NC-PEG$\mathrm{COOH}$, that could be used for further conjugation.

The influence of 3 different $\mathrm{pH}$ environments on $\mathrm{NC}$ fluorescence, along with untreated $\mathrm{NC}$ and $\mathrm{RB}$ alone is shown on Fig. 1, $B$ and normalized fluorescence (accounting for NC dilution) is shown on Fig. 1, $C$.

Available protocols propose using basic $\mathrm{pH}$ $(>12)$ for conjugation, however this condition can degrade the used NC (and is potentially harmful for many other NC). However, at pH 8 we observed almost complete preservation of fluorescence (Fig. 1, $B$ and $C$ ).

\section{Protein conjugation to NC-PEG-COOH.}

6. An equal volume of 0.1 M MES (2-(N-Morpholino)ethanesulfonic acid hydrate) buffer, $\mathrm{pH} 5$ was added to NC-PEG-COOH obtained in step 5.

7. Lectin ConA was dissolved in 0.1 M MES buffer, $\mathrm{pH} 5$ (the conjugation rate: $2 \mathrm{mg}$ of protein $/ 1 \mathrm{ml}$ of NC-PEG-COOH, the concentration of NC: $0.02 \mathrm{mg} / \mathrm{ml})$.

8. Lectin ConA was mixed with NC-PEG$\mathrm{COOH}$ and then dry EDC, MW 191.7, (Sigma-Aldrich) was added to obtain a $0.25 \mathrm{M}$ solution.

9. The reaction lasted $2 \mathrm{~h}$ at room temperature, with mixing.

10. Dialysis against $10 \mathrm{mM}$ Tris, $\mathrm{pH} 6.8$ was performed. The final volume of NC-PEG-CO-NHprotein was $4 \mathrm{x}$ the initial NC-PEG volume, (that is, i.e., fluorescence and concentration of $\mathrm{NC}$ in the final sample was $0.25 \mathrm{x}$ the initial concentration of NC-PEG used in the reaction at step 2). If needed, preservative compounds of choice can be added at this step.

Application of conjugated NC. The obtained NC-PEG-CO-NH-ConA conjugates were tested for specific interaction with living and necrotic human HeLa cells. Conjugation with protein resulted in a slight increase in size of NC-PEG-CO-NH-protein complexes (Fig. 2, B) vs unconjugated NC-PEG (Fig. 2, A); those complexes represented a small percentage of NC manifested aggregation. Still both NC-PEG and NC-PEG-protein demonstrated a prominent Brownian motion, and were detected due to bright point spread function of initial point objects using a cooled Zeiss CCD camera; this did not make possible the direct measurement of the size of NCPEG-protein, but we estimated it as 0.1 to $0.2 \mu \mathrm{m}$ for NC-PEG-CO-NH-ConA basing on the behavior of particles with known sizes. Incubation of NC-PEGCO-NH-ConA with viable cells demonstrated no staining (in fact, there was negative contrasting of viable cells with intact membrane vs positive background due to unbound NC-PEG-CO-NH-ConA fluorescence), as shown in Fig. 2, $C$ (differential interference contrast [DIC] microscopy) and Fig. 2, $D$ (fluorescence). At the same time, treatment of necrotic HeLa cells allowed effective staining on intracellular compartments with NC-PEG-CO-NHConA, as shown in Fig. 2, E (DIC) and Fig. 2, F (fluorescence), clearly demonstrating accumulation of conjugates on the surface and inside dying cells.

Conclusions. Here we propose the optimized protocol for a covalent coupling of proteins to NCPEG. The method ensures removal of chemical byproducts by dialysis, and it results in a minimal final dilution of NC-protein conjugates (4x dilution). The proposed approach omits the need for centrifugation of small NC, which is usually a limiting step due to the needed high accelerations of $>20000 \mathrm{~g}$. Besides, it allows the $\mathrm{pH}$ of the reaction mixture to be maintained in the range between 6 to 8 , preserving the properties of both protein and NC. The proposed approach can be easily modified for covalent targeting of different PEG-bearing nanocomposites.

\section{Acknowledgements}

This work was supported by Grants from the Ministry of Healthcare of Ukraine (project 0116U000759). We thank are thankful to Cedars-Sinai Medical Center's International Research and Innovation in Medicine Program, and the Association for Regional Cooperation in the Fields of Health, Science and Technology (RECOOP HST Association) for their support. MB acknowledges the National Science Centre for its financial support within the Sonata 8 project no. UMO-2014/15/D/ST5/02744.

\section{References}

1. Gerion D, Pinaud F, Williams SC, Parak WJ, Zanchet D, Weiss S, Alivisatos AP. Synthesis and properties of biocompatible water-soluble silica-coated CdSe/ZnS semiconductor quantum dots. J Phys Chem B. 2001; 105(37): 8861-8871.

2. Rogach AL, Nagesha D, Ostrander JW, Giersig M, Kotov NA. "Raisin bun"-type composite spheres of silica and semiconductor nanocrystals. Chem Mater. 2000; 12(9): 2676-2685.

3. Medintz IL, Uyeda HT, Goldman ER, Mattoussi H. Quantum dot bioconjugates for imaging, labelling and sensing. Nat Mater. 2005; 4(6): 435-446. 

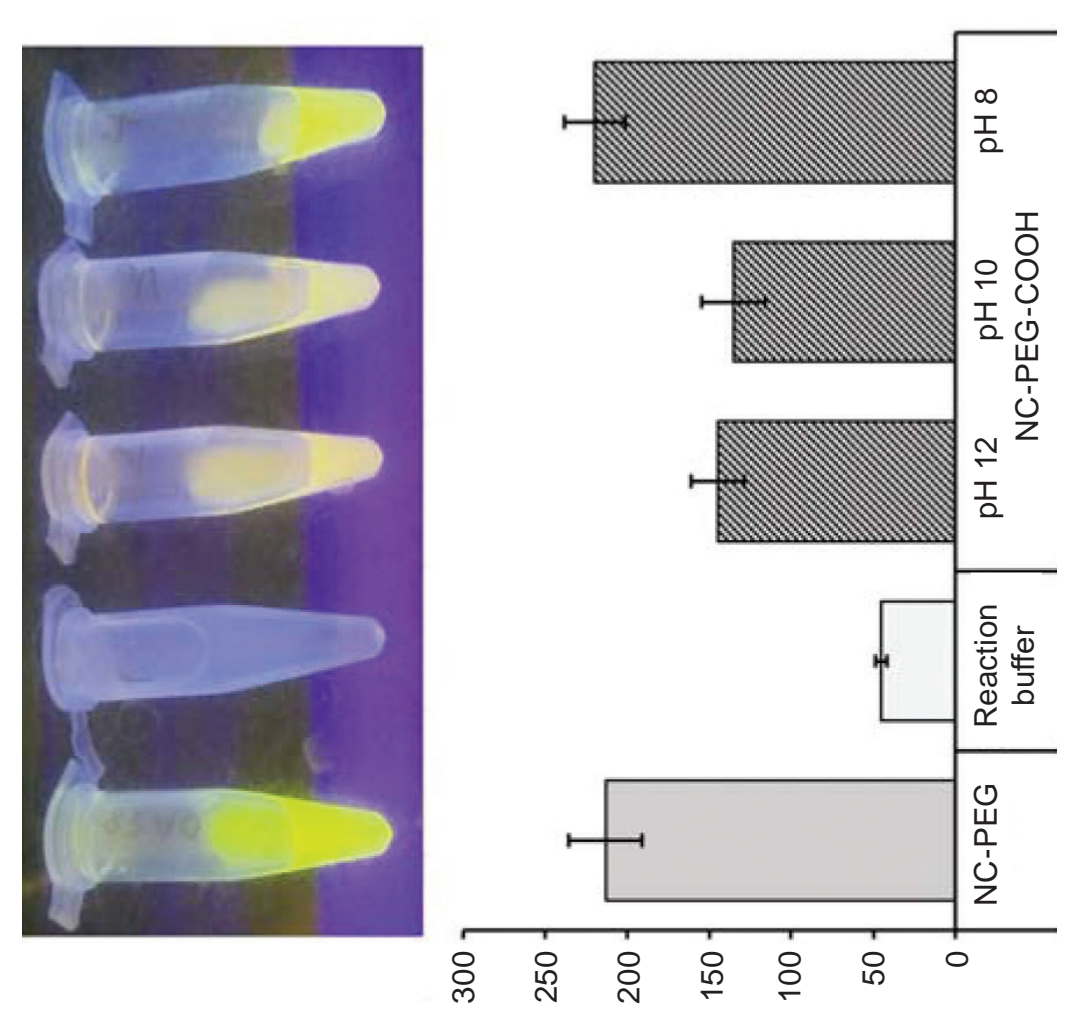

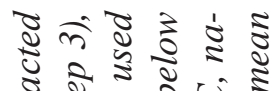
के के

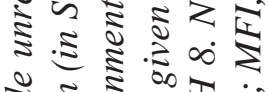

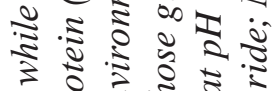

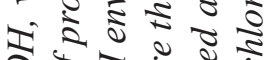
ठิ บิ पि के

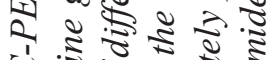
之. है ४ के है है : ระ.

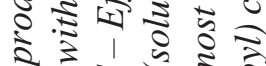
$\therefore$ प 전 บั

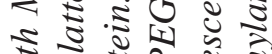

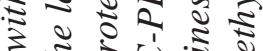
ปั

$\infty$

u

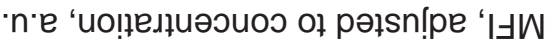

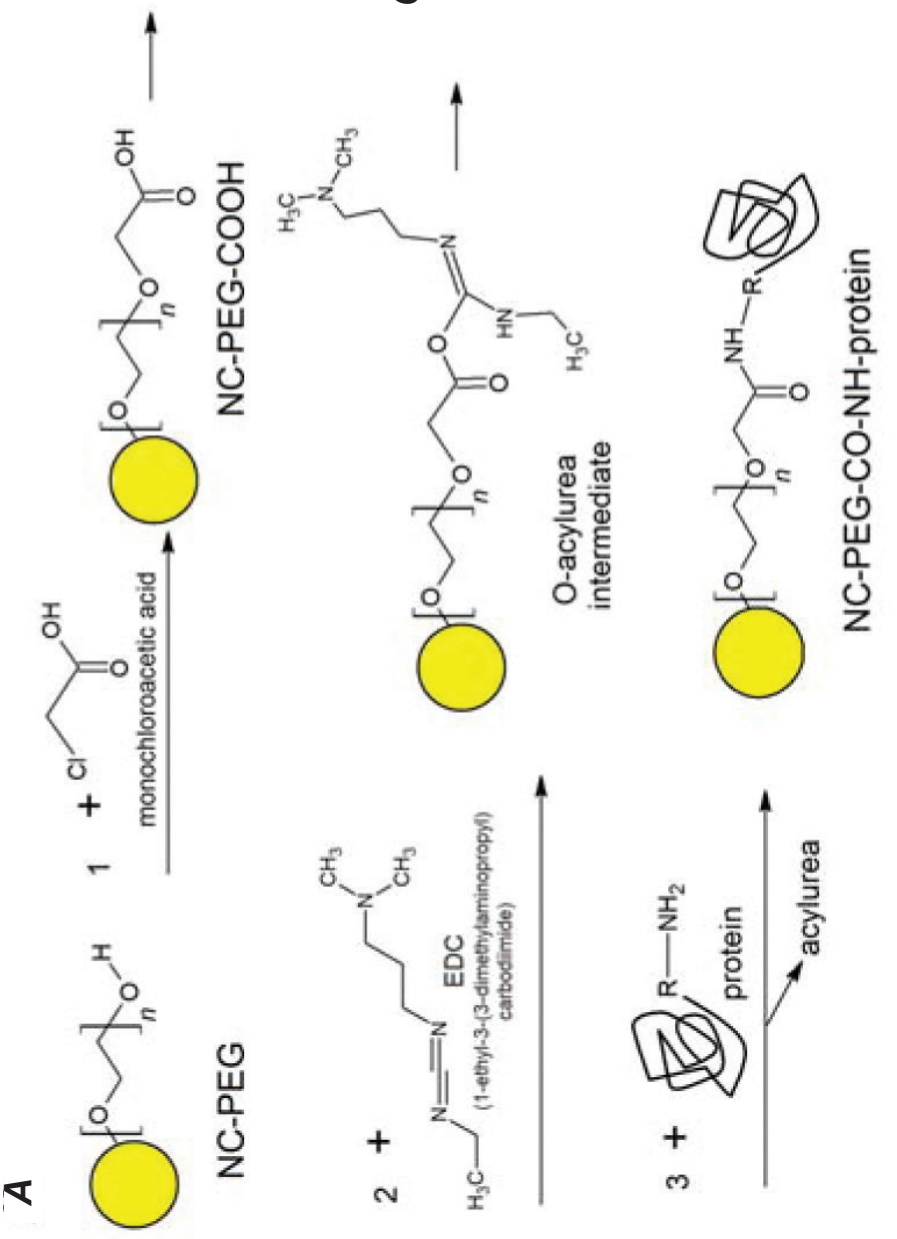

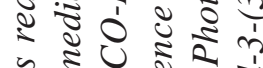

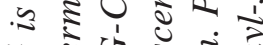
৬)

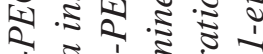

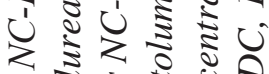
1 )

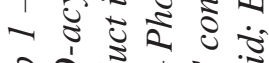
ब्व

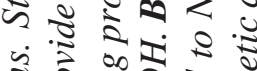
ฉ 造 5. रำ $\therefore$ 过

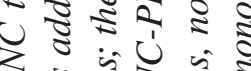
$z y$ y - U 吾䓃吾

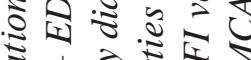

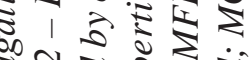
डิ वे के वे

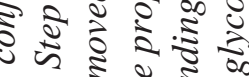
นิ ज्ञ क्षे के

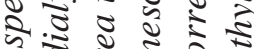
के $\supset$ है

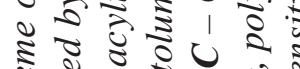

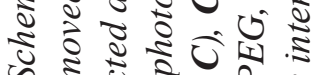

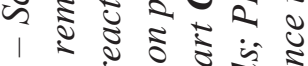

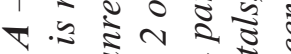
- 45 क 5

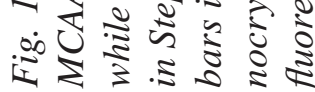




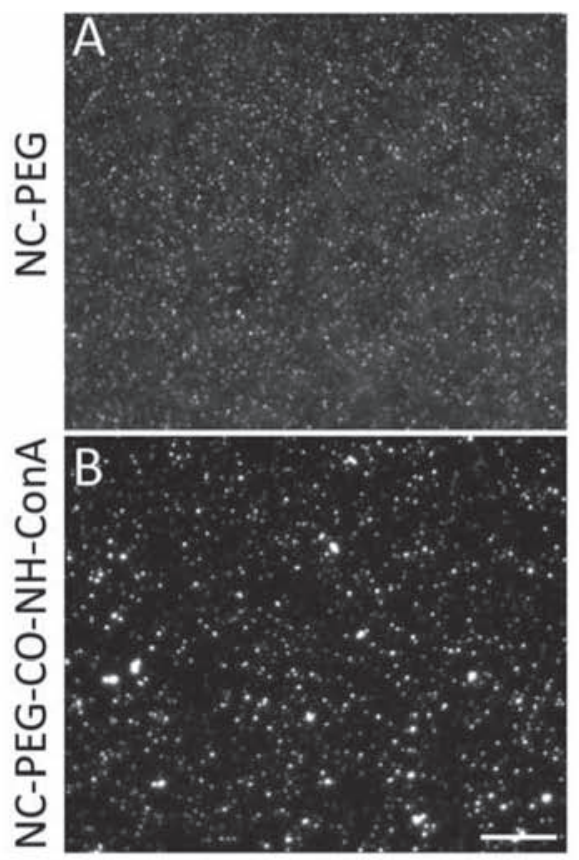

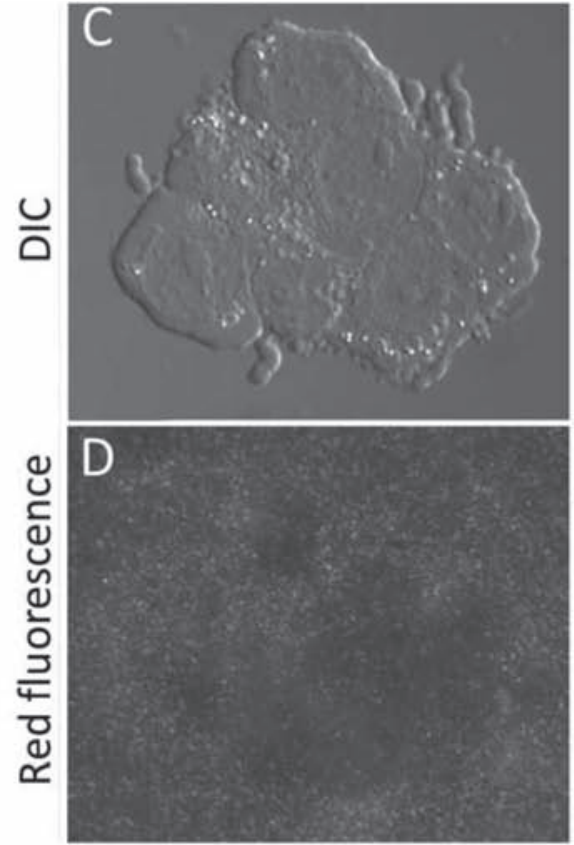

Viable
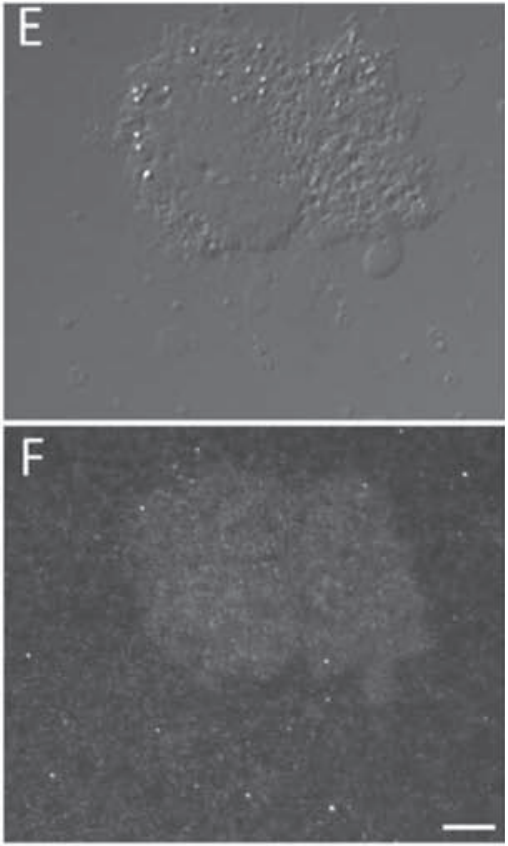

Necrotic

Fig. 2. Targeting intracellular glycans of HeLa cells with created NC-PEG-CO-NH-ConA. $\boldsymbol{A}-$ NC-PEG (before protein conjugation step) and $\boldsymbol{B}-N C-P E G-C O-N H$-ConA (after protein conjugation step). NC-PEG$\mathrm{CO}-\mathrm{NH}$-ConA complexes were incubated with viable $(\boldsymbol{C}, \boldsymbol{D})$ or necrotic $(\boldsymbol{E}, \boldsymbol{F}) \mathrm{HeLa}$ cells. Fluorescent $(\boldsymbol{A}, \boldsymbol{B}$, $\boldsymbol{D}, \boldsymbol{F})$ and DIC $(\boldsymbol{C}, \boldsymbol{E})$ microscopy. Scale bar equals $5 \mu \mathrm{m}$. NC, nanocrystals; PEG, polyethylene glycol; DIC, differential interference contrast

4. Bharti S, Kaur G, Gupta S, Tripathi SK. PEGylation of CdSe/ZnS core/shell nanoparticles and its behavior at different pH. J Lumin. 2017; 181: 459-466.

5. Zhang P, Han H. Compact PEGylated polymercaged quantum dots with improved stability. Colloids Surf A Physicochem Eng Asp. 2012; 402: 72-79.

6. Zhao H, Yang K, Martinez A, Basu A, Chintala R, Liu HC, Janjua A, Wang M, Filpula D. Linear and branched bicin linkers for releasable PEGylation of macromolecules: controlled release in vivo and in vitro from mono- and multi-PEGylated proteins. Bioconjug Chem. 2006; 17(2): 341-351.

7. Hermanson G. Bioconjugate Techniques. 3rd ed. London: Academic Press; 2013. 1200 p.

8. Bilyy R, Podhorodecki A, Nyk M, Stoika R, Zaichenko A, Zatryb G, Misiewicz J, Strek W. Utilization of $\mathrm{GaN}: \mathrm{Eu}^{3+}$ nanocrystals for the detection of programmed cell death. Phys E Low Dimens Syst Nanostruct. 2008; 40(6): 20962099.
9. Sojka B, Podhorodecki A, Banski M, Misiewicz J, Drobczynski S, Dumych T, Lutsyk MM, Lutsyk A, Bilyy R. $\beta$-NaGdF4:Eu ${ }^{3+}$ nanocrystal markers for melanoma tumor imaging. RSC Adv. 2016; 6(63): 57854-57862.

10. Dumych T, Lutsyk M, Banski M, Yashchenko A, Sojka B, Horbay R, Lutsyk A, Stoika R, Misiewicz J, Podhorodecki A, Bilyy R. Visualization of melanoma tumor with lectin-conjugated rare-earth doped fluoride nanocrystals. Croat Med J. 2014; 55(3): 186-194.

11. Plotz PH, Rifai A. Stable, soluble, model immune complexes made with a versatile multivalent affinity-labeling antigen. Biochemistry. 1982; 21(2): 301-308.

12. Bilyy RO, Shkandina T, Tomin A, Muñoz LE, Franz S, Antonyuk V, Kit YY, Zirngibl M, Fürnrohr BG, Janko C, Lauber K, Schiller M, Schett G, Stoika RS, Herrmann M. Macrophages discriminate glycosylation patterns of apoptotic cell-derived microparticles. J Biol Chem. 2012; 287(1): 496-503. 